\title{
LOS CICLOS DE COMPETENCIAS PARA EL DesarRollo de procesos de COOPERACIÓN LOCAL
}

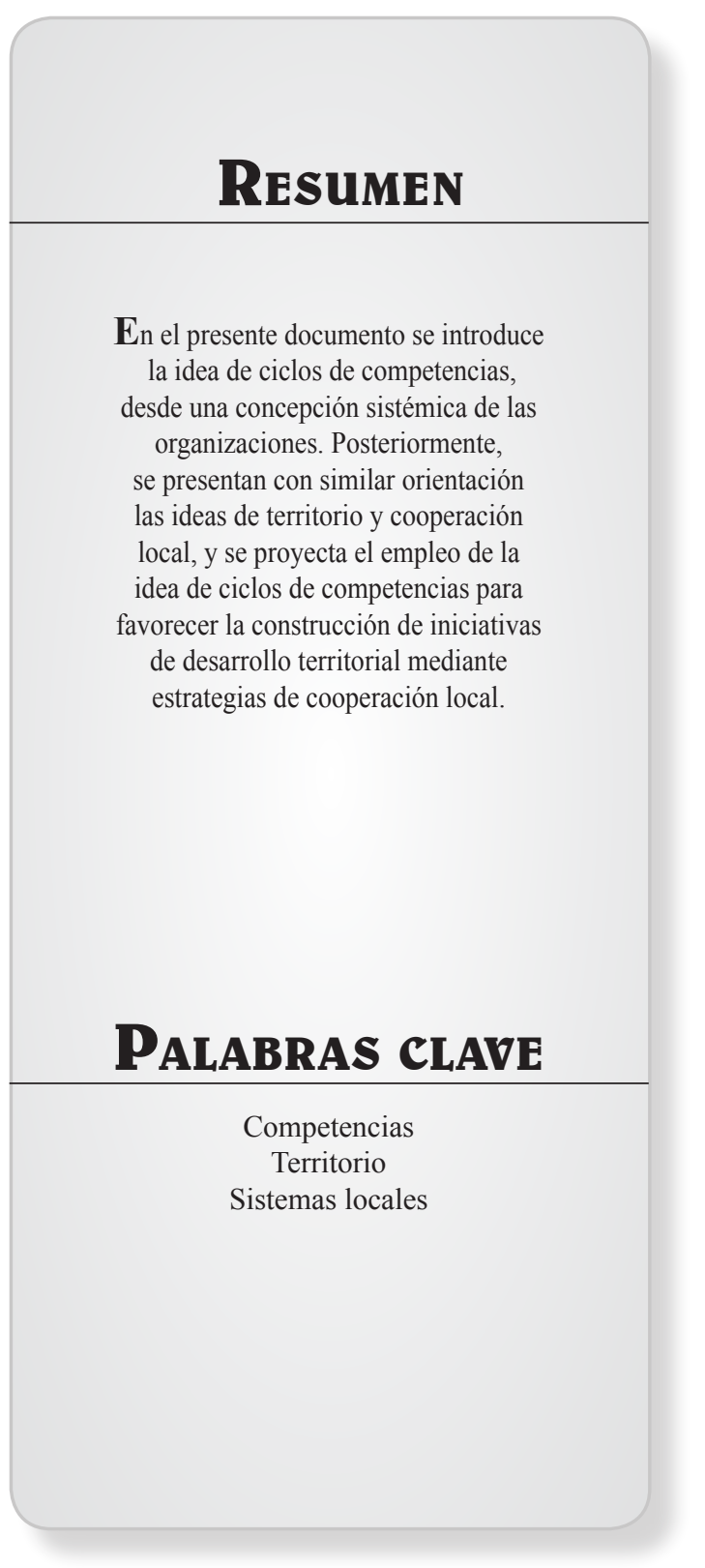

María Alejandra Rubiano Navas*
Iván Montoya Restrepo**
Luz Alexandra Montoya Restrepo***

\section{LAS ORGANIZACIONES COMPRENDIDAS COMO SISTEMAS}

$\mathbf{A}$ identifican diferentes especies de organizaciones en diferentes clases de ambientes o entornos. La teoría organizacional parece pasar de ser mecanicista a organicista, en donde las relaciones y diferencias entre moléculas, células, organismos, especies y ecología, son paralelas a aquellas entre individuos, grupos, organizaciones, poblaciones (especies) y su ecología social, mediante la herramienta de la metáfora. (Morgan, 1996; Montoya, Alexandra, 1999).

Gracias a esta herramienta de reflexión, resulta posible estudiar la organización orgánicamente, identificando sus necesidades como sistemas abiertos, los procesos de adaptación de las organizaciones al entorno, el ciclo de vida

\footnotetext{
* Ingeniera Agrónoma, Universidad Nacional de Colombia.

** Profesor Asociado, Facultad de Agronomía Universidad Nacional de Colombia.

*** Profesora Asociada, Facultad de Ciencias Económicas,

Universidad Nacional de Colombia.
} 
organizacional, los factores que influyen en la salud y el desarrollo de las organizaciones y las relaciones entre las especies y su ecología; de tal manera que los objetivos, estructuras y eficacia, pasan a ser subsidiarios ante los problemas de supervivencia y otras necesidades nuevas.

Entonces, las organizaciones pueden ser presentadas como sistemas vivientes o conjuntos de sistemas que muestran órdenes más elevados que los de sus componentes, siendo interpretado el orden en términos de gran complejidad y determinación consciente para moverse hacia objetivos autoestablecidos.

De acuerdo con Montoya y León, (Op. Cit.) las organizaciones al igual que los individuos, tienen necesidades y existe una compatibilidad potencial entre las de la organización y las individuales. La mayor efectividad se logrará en la medida en que la organización se estructure de manera que las personas que la componen puedan satisfacer a través de su trabajo las necesidades propias y las de la empresa. Esto es posible, empleando la jerarquización de las necesidades individuales propuesta por Maslow, para hacer una jerarquización similar aplicada a las organizaciones. Por lo anterior, es viable combinar las propuestas de necesidades de Neff, Maslow y Fromm con la pirámide invertida de la organización, en donde se satisfagan las necesidades esenciales del hombre para su mayor motivación dentro de la empresa $y$, al mismo tiempo, esta logre mayores niveles de productividad y competitividad, basados en una mayor efectividad, como se observa en la figura 1. En el cuadro 1, se explica brevemente la interpretación de la propuesta.

\section{Abstract}

In the document is introduced the idea of cycles of competeces, from a systemic conception of the organizations. After this introduction, it is presented with similar orientation a reconsideration of the notions of territory and local cooperation,

and is projected the employment of the idea of cycles of competences in order to favor the construction

of initiatives of territorial development by means of strategies of local cooperation.

\section{Key Words}

\author{
Competences \\ Territory \\ Local systems
}


Figura 1

Esquema de necesidades combinando los planteamientos de Abraham Maslow, Max Neff y Erich Fromm

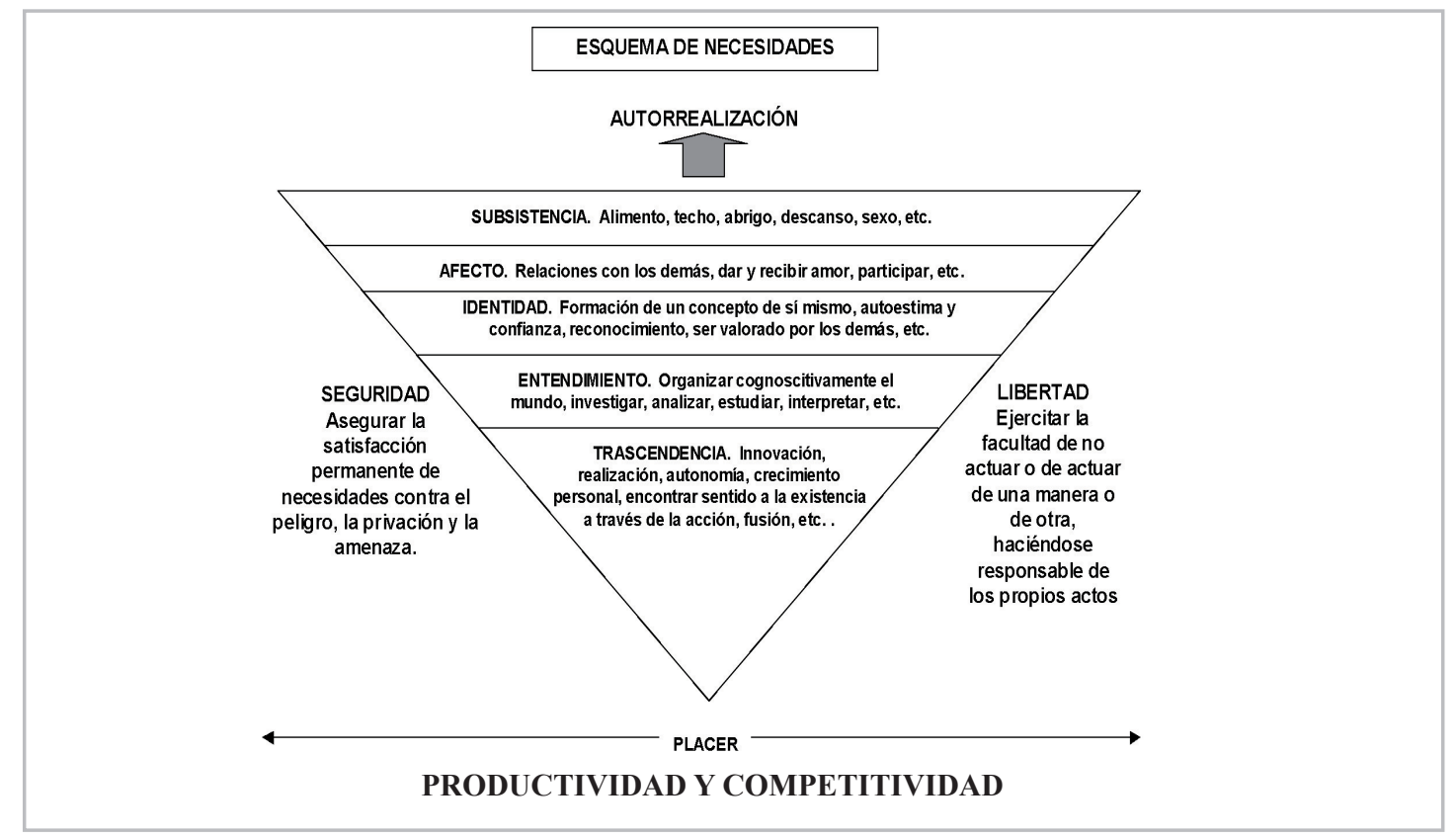

Fuente. Montoya y León

Cuadro 1.

Paralelo necesidades humanas con necesidades de la organización

\begin{tabular}{|c|c|}
\hline Necesidad humana & Necesidad de la organización \\
\hline Subsistencia & Demanda \\
\hline Afecto & Recursos \\
\hline Identidad & Dirección \\
\hline Entendimiento & Eficiencia \\
\hline Trascendencia & Eficacia \\
\hline
\end{tabular}

Sustentadas en:

Seguridad y libertad individual y organizacional

Producirán placer y autorrealización en los individuos y productividad y competitividad en la organización. 
Cuando se reconoce que individuos, grupos y organizaciones tienen necesidades que deben satisfacerse, la atención se dirige invariablemente al hecho de que dependen de un amplio entorno para sus variados tipos de sustento. A nivel práctico, los sistemas abiertos se centran en una serie de puntos clave: hacer hincapié en el entorno en el cual la organización existe; definir una organización en términos de subsistemas interrelacionados; intentar establecer sistemas e identificar y eliminar disfunciones potenciales -el principio de diferenciación y de integración es muy útil para organizar las diferentes tareas dentro de la misma organización-.

\section{De una visión estática a una adAPTATIVA PARA ORGANIZACIONES}

$\mathbf{E}$ teórico que considera los fenómenos orgánicos e inorgánicos encajados en estructuras con su ambiente en movimiento. Las organizaciones, entendidas como sistemas abiertos ${ }^{1}$, deberían involucrar como sistema social las tareas, las interacciones y los sentimientos, en relación con un ambiente dinámico (Checkland, 1.994). Kast y Rosenzweig consideran que el sistema organizacional está conformado por subsistemas: tecnológico, de metas y valores o estratégico, psicosocial o humano/cultural y estructural, en contraposición a la teoría clásica. La intersección de estos subsistemas, que se encuentran inmersos en intercambios de energía, materiales e información, es el subsistema administrativo o de dirección (Kast y Rosenzwieg, 1.979, Montoya, I. 1.999).

Figura 2

La organización como sistema cerrado en la Teoría Clásica

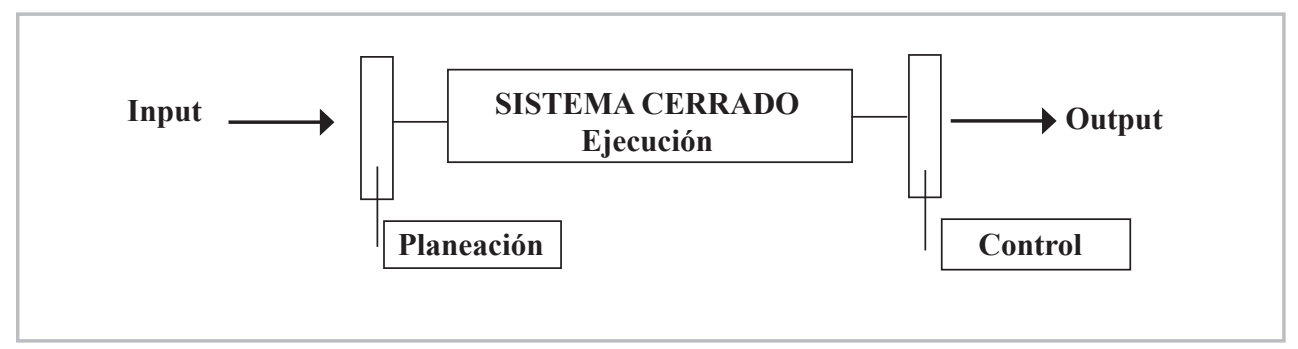

\footnotetext{
${ }^{1}$ La Teoría de la Dependencia (adaptación de la organización al entorno), manifiesta que "las organizaciones son sistemas abiertos que necesitan gestionar cuidadosamente, satisfacer y equilibrar sus necesidades internas y adaptarse a las circunstancias ambientales o del entorno". "No hay un único modo de organización. La forma apropiada depende de la clase de tarea o entorno con el que se está relacionando" (Morgan, 1996).
} 
Figura 3

Sistema organizacional de Kast y Rosenzweig adaptado. Modelo de sistema abierto

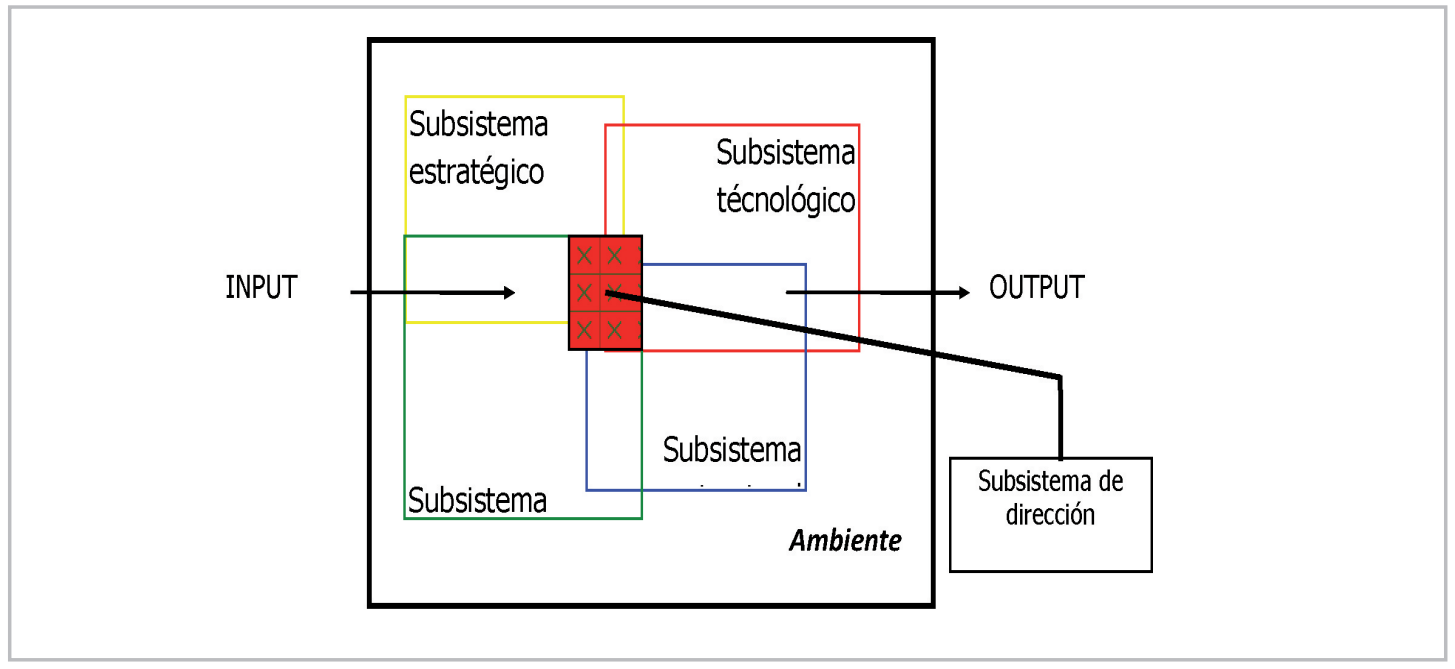

Fuente. Tomado de Montoya, I.1999

El enfoque de sistemas permite analizar la interacción de la organización con el ambiente. Así, se presenta una continua evolución que contribuye al control de variables para reprogramar el rumbo de la organización, de allí se origina el concepto de retroalimentación (Kast y Rosenzweig, 1.979). Estas ideas han mostrado el camino a las teorías de organización y de gestión, de forma que se involucren en el análisis los requerimientos del entorno.

Lo más interesante de este enfoque también compartido $^{2}$ por Charles Perrow, Talcott Parsons ${ }^{3}$ y otros, es el análisis que puede hacerse de la interacción del sistema con el ambiente. Así se presenta una continua evolución que contribuye sin lugar a dudas al control de variables para reprogramar el rumbo de la organización, de allí el más relevante concepto del enfoque: la retroalimentación ${ }^{4}$.

Lawrence y Lorsch proponen una teoría contingente de la organización según la eficacia que puede lograrse; encontrarían una relación inversa entre diferenciación e integración en relación con el ambiente, valga decir, entre mayor es la diferenciación del contexto, los planes de integración están más asociados ${ }^{5}$. El estructural funcionalismo, en asocio con la teoría de sistemas, sostiene que todas las organizaciones poseen elementos comunes en su estructura y que la sociedad es un sistema social. De esta manera se integran los conceptos de sistema social y organizacional.

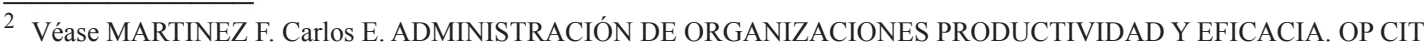
P.P.297-318.

3 Véase PARSONS, T. \& PERROW, C. COMPLEX ORGANIZATIONS. 2nd. Edition. Scott and Foresman Ed. Glenview, Illinois, US. 1979.

4 Véase KAST \& ROSENZWEIG. OP CIT pp.131-132.

5 Véase LAWRENCE, Paul \& LORSCH, Jay . ORGANIZACIÓN Y AMBIENTE. Editorial Labor, Barcelona, 1973. P.P.111-115.
} 
Un hipótesis para la aproximación a lo adaptativo, permite considerar la problemática de la organización como su capacidad para autoproducirse en un entorno cambiante. Además, la empresa tiene dos necesidades adicionales consistentes en ajustar sus estructuras hacia un esfuerzo de autoproducción por una parte, mientras que por otra requiere aprender y desarrollarse cuando se apropian los procesos de conversión de estructuras para garantizar la autoproducción de sí misma (Montoya, I. 1999); si la empresa posee estas capacidades, que también equivalen a la permanencia, el crecimiento y la rentabilidad, será competitiva. La consideración inicial reside en la suposición de que se pueden entender las organizaciones como organismos "vivos" en el sentido de que ejercen presión por su subsistencia, mientras que deben adaptar sus estructuras internas (como sistema abierto) y generan un aprendizaje originado en el proceso de cognición al lograr las dos metas recién mencionadas (la supervivencia y la renovación de las estructuras); con lo cual puede empezar a tener conciencia el sistema- organización. Esta conciencia bien puede entenderse como el aprendizaje organizacional y tecnológico. (Montoya, I. 1999)

Cuadro 2

Metáfora dinámica de las necesidades de las organizaciones empresariales

\begin{tabular}{|c|c|c|}
\hline Autoproducción de sí, & $\begin{array}{l}\text { Conversión de estructuras en } \\
\text { relación con el medio ambiente. }\end{array}$ & $\begin{array}{l}\text { Procesos de aprendizaje organi- } \\
\text { zacional. }\end{array}$ \\
\hline Permanencia o pervivencia & $\begin{array}{l}\text { Crecimiento (Mejora de las } \\
\text { estructuras entre elementos). }\end{array}$ & Rentabilidad. \\
\hline Direccionamiento estratégico. & Gerencia del día a día. & Cultura. \\
\hline $\begin{array}{l}\text { Mejorar velocidad de entrada de } \\
\text { los ingresos ocasionados por } \\
\text { ventas. }\end{array}$ & $\begin{array}{l}\text { Mejora en el manejo de los inven- } \\
\text { tarios y el dinero inmovilizado } \\
\text { dentro del sistema. }\end{array}$ & $\begin{array}{l}\text { Mejora en los costos deoperación } \\
\text { y en los que se incurre para } \\
\text { convertir el dinero inmovilizado } \\
\text { en ingresos por ventas. }\end{array}$ \\
\hline $\begin{array}{l}\text { Problema del autocontrol } \\
\text { (Ackoff). }\end{array}$ & $\begin{array}{l}\text { Problema de la Humanización } \\
\text { (Ackoff). }\end{array}$ & $\begin{array}{l}\text { Problema de la Ambientalización } \\
\text { (Ackoff). }\end{array}$ \\
\hline $\begin{array}{l}\text { Problemática de la generación } \\
\text { de competencias }\end{array}$ & $\begin{array}{l}\text { Problemática de la innovación y } \\
\text { el cambio estructural }\end{array}$ & $\begin{array}{l}\text { Problemática del aprendizaje y } \\
\text { acumulación de rutinas }\end{array}$ \\
\hline
\end{tabular}

Fuente. Montoya, I. 1999 con revisión posterior. 
El proceso debe realizarse a manera de espiral, de forma tal, que la cultura y los aprendizajes tecnológicos y organizacionales se traduzcan en la generación de nuevas posibilidades para la permanencia de la empresa en el mercado. Estas posibilidades son competencias, o capacidades de actuar de la organización en una situación problemática particular dentro de un contexto preciso cambiante. Tales competencias vienen a hacerse instrumentales como conocimientos que se expresan en rutinas estandarizadas o bien conocidas cuando la organización confronta un reto particular en el mercado.
La permanencia de la organización en el mercado posibilita los procesos de adaptación que conducen a los cambios estructurales en procesos y activos, así como en la identificación de productos y servicios, que favorecen un programa de gerencia de la innovación. Por último, la innovación permite acumular experiencias que se traducen en aprendizajes tecnológicos y sociales, los cuales se convertirán en el futuro en nuevas competencias. (Montoya y León, Op. Cit)

\section{LOS CICLOS DE COMPETENCIAS}

$\mathbf{p}$

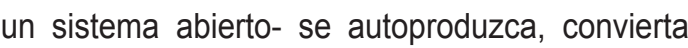
sus estructuras y aprenda, con el propósito de ser competitiva, deberá establecer una serie de competencias centrales que constituyen una ventaja competitiva que se construye con el tiempo y que no se puede imitar fácilmente (LeonardBarton, 1995; Prahalad, 1998, citados en Montoya y León, Op. Cit.).

Para crear y mantener competencias centrales se requieren dos habilidades básicas dentro de una organización (Leonard-Barton, 1995):

- Saber cómo administrar las actividades que crean conocimiento.

- Entender exactamente qué constituye una competencia central, cuáles son sus dimensiones.
De acuerdo con Leonard y Barton, existen cuatro actividades críticas en la construcción de conocimiento, básicas para la gerencia de la innovación (Leonard y Barton, 1995):

- Conseguir cómo resolver problemas operativos a través de experticia multifuncional.

- Integrar nuevas herramientas y metodologías.

- Experimentar y crear prototipos.

- Importar conocimiento externo a la organización y aprender del mercado.

Según Hamel y Prahalad, y con el propósito de evitar la aparición de rigideces centrales, una organización innovadora deberá crear, mantener y mejorar un ciclo de competencias que le permitirán sostenerse en un mercado cada día más globalizado. Este ciclo está conformado por 
las competencias centrales -core competencies o core capabilities- de la organización, la gerencia de la innovación y el aprendizaje organizacional en donde, inicialmente, la empresa deberá identificar sus competencias centrales, transformarlas en nuevos procesos, productos $y / 0$ servicios $y$, finalmente, deberá aprender de los proyectos afortunados o desafortunados, utilizando esta experiencia para mejorar las competencias existentes o para desarrollar nuevas competencias (Tidd, 2.000). Este ciclo responde de manera muy precisa a las necesidades de una metáfora adaptativa de las organizaciones.

Figura 4

El ciclo de las competencias

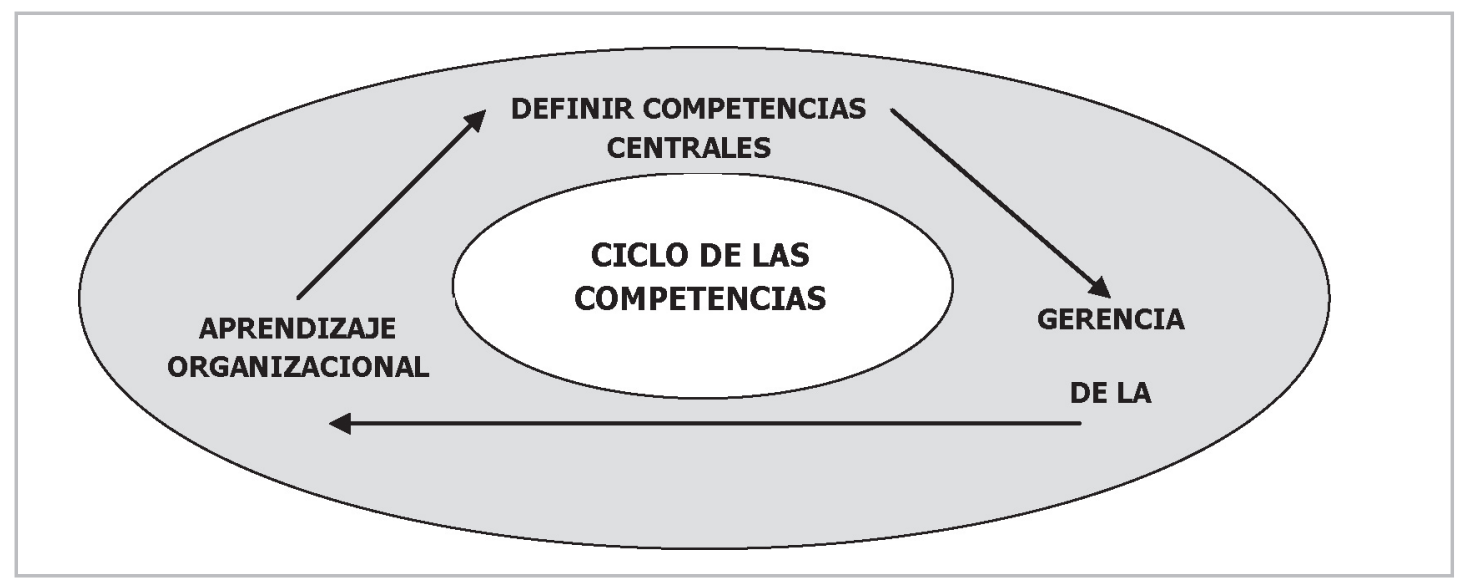

Fuente. Tidd, J. 2000

La organización basada en las competencias medulares o centrales, no debería reemplazar a la empresa tradicional; sin embargo, la generación de una ventaja competitiva sostenible en las compañías no residirá en sus productos, sino en sus competencias centrales ${ }^{6}$ : en la capacidad para gerenciar las tecnologías y las habilidades productivas dentro de los saberes individuales de todas las unidades del negocio, adaptándose rápidamente al cambio (Tidd, 2.000).

Para que las competencias puedan ser convertidas en nuevos productos, procesos y/o servicios, se requiere definir una estrategia de innovación y los planes de inversión en investigación y desarrollo, procesos de innovación tecnológica, patentes, nuevos productos, procesos o servicios, entre otros, aspectos básicos de la gerencia de la innovación. La organización deberá identificar las principales fuentes de oportunidades tecnológicas, representadas en: avances científicos, avances tecnológicos en otras industrias relacionadas y retroalimentación positiva de estos avances para su beneficio. (Montoya y León, Op. Cit)

\footnotetext{
${ }^{6}$ Las competencias centrales requieren un enfoque altamente estratégico -no se trata de formular 20 o 30 competencias, sino las que sean estrictamente necesarias para el negocio. Esto dependerá de la arquitectura estratégica, que permitirá identificar las competencias futuras de mayor relevancia para el éxito de la organización (Tidd, 2.000).
} 
Es allí donde la gerencia de conocimiento permitirá monitorear la información y el conocimiento producidos en la organización o en el medio, apropiarse de ese conocimiento, acumularlo y crear nuevo, generando un proceso de aprendizaje organizacional constante, que se reflejará en el éxito de la empresa en sí misma y en el mercado y conducirá al fortalecimiento de sus competencias centrales y de la memoria organizacional (Pitt y Clarke, 1.999; Tidd, 2.000).

Figura 5

Componentes del aprendizaje organizacional

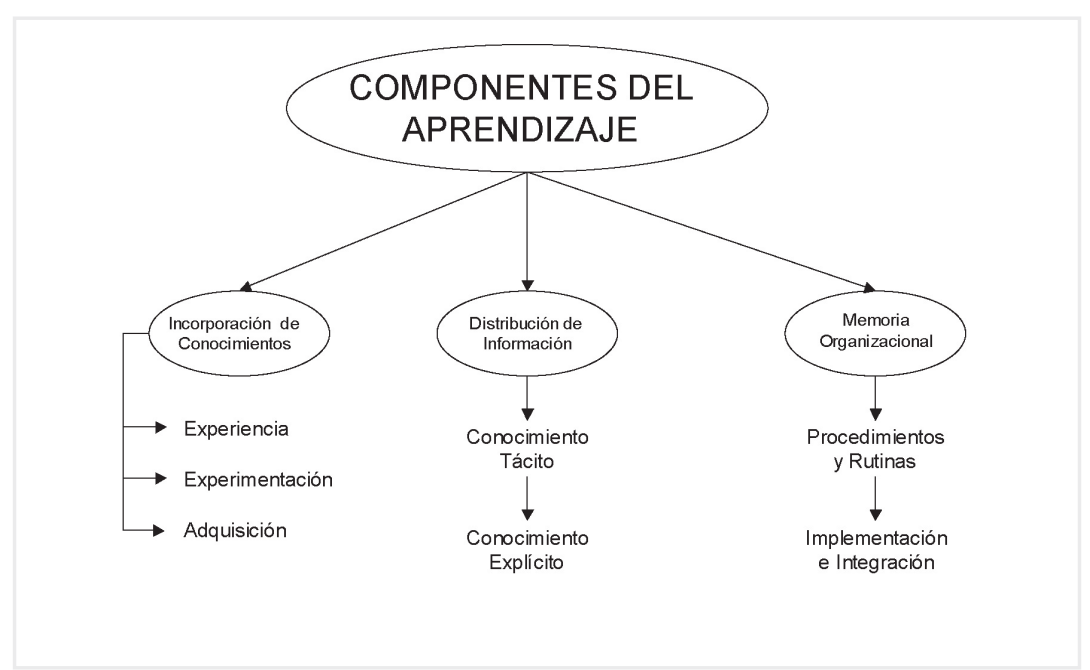

Fuente. Tomado de Montoya y León, Op. Cit.

Las organizaciones pueden apropiar conocimiento a través de la experiencia, la experimentación o la adquisición. El aprendizaje por la experiencia es el menos efectivo, debido a que, en la práctica, las organizaciones no trasladan fácilmente la experiencia en nuevo conocimiento; además, el aprendizaje puede ser conseguido sin intención o puede no resultar en el mejoramiento de la efectividad.

La experimentación es un enfoque más sistemático de aprendizaje. Esta es una característica central de las actividades de I\&D, investigación de mercados y algunas alianzas organizacionales -aunque el uso de alianzas para el aprendizaje es menos común y requiere la intención de utilizarlas como una oportunidad de aprendizaje, receptividad de know how externo y transparencia suficiente en las asociaciones-.

La adquisición de know how se convertirá en aprendizaje organizacional, dependiendo de la forma en que se realice dicha adquisición y del proceso de transferencia de tecnología.

En resumen, el ciclo de competencias inicia con la identificación y/o planteamiento de las competencias centrales de la organización, mediante el estudio del estado deseado y el actual. El contraste de estos dos estados -o selección de alternativas- permitirá definir la forma como la organización debe adquirir nuevas competencias o 
fortalecer las existentes. Después de precisar las opciones más viables, la organización establecerá las variables, áreas o procesos críticos para llevar a cabo innovaciones (gestión de personal, gestión de calidad, gestión de mercadeo, gestión del servicio, gestión ambiental, etc), dentro de las dimensiones instrumental, actitudinal y organizacional, e implementará una serie de planes de acción con el objeto de rediseñar o reestructurar su forma de operar tanto interna como externamente. Una vez implementados los cambios o mejoras, la organización evaluará las capacidades de gestión del conocimiento desarrolladas (capacidad de captar información, analizarla y generar conocimiento, conservarlo, difundirlo, protegerloy normalizarlo), analizando el grado de aprendizaje organizacional, y reiniciará el ciclo teniendo en cuenta los resultados obtenidos y las competencias centrales planteadas. Este proceso hará que la organización trabaje en función de sus competencias centrales, minimizando costos y maximizando su productividad y competitividad, a través del conocimiento. (Montoya y León, op. Cit)

\section{El CONCEPTO de TeRRITORIO}

\section{$\mathbf{L}$} modificado a lo largo de la historia, de acuerdo con la comprensión de la complejidad de sus relaciones e interacciones y con los cambios y evoluciones propios de las sociedades rurales. En el presente trabajo se presenta el territorio, como una categoría "...llamada a sintetizar, en un marco coherente de interpretación y gestión, muchos de los elementos que constituyen las nuevas estrategias de desarrollo rural..." (Echeverri y Ribero, 1998; citado en Rubiano, 2005).

Uno de los desafíos más grandes del desarrollo rural, ha sido el hecho de redescubrirse y redefinir sus políticas permanentemente, el hacia dónde, desde dónde, quiénes y qué transformar en la sociedad rural, se han convertido en las preguntas frecuentes y periódicas a lo largo de la historia. Desde luego, estas preguntas han tenido que ser resueltas de acuerdo con los espacios temporales, con los efectos de las transformaciones realizadas con anterioridad, y con las formulaciones ideológicas del momento, siempre buscando el desarrollo en los distintos escenarios. (Rubiano, 2005). Esta situación ha dado origen a cambios y movimientos sistemáticos a manera de oleadas en las estructuras ideológicas, políticas y en las realidades locales del sector rural. Sin embargo, dichos cambios en las estructuras ideológicas y el ritmo de estos en las realidades locales, han tenido brechas temporales las cuales han dificultado la posibilidad de comprender prontamente de dónde provienen y de esta manera asimilar, expresar y responder desde los escenarios rurales a dichos cambios. (Plaza, 2002)

La noción de desarrollo involucra teorías de cambio social, esto significa que las diferentes interpretaciones sobre el desarrollo están relacionadas directamente con posiciones ideológicas las cuales se cruzan con nociones de tiempo específicas (Plaza, 2002). El desarrollo rural ha establecido a lo largo de la historia y del tiempo dos vertientes ideológicas, las cuales han determinado múltiples paradigmas que van desde lo económico productivo, hasta lo 
socio-cultural. Estos cambios en las estructuras ideológicas del desarrollo rural han estado sujetos a una temporalidad definida. Durante las últimas cinco décadas se han desarrollado una serie de temas dominantes o paradigmas (cómo algunos autores lo referencian), que han marcado los cambios, el hacia dónde se dirigen y a quiénes o qué benefician. El siguiente cuadro establece las principales ideas, políticas o prácticas que han primado para el desarrollo rural desde la década de los 50 hasta la actualidad. El cuadro no hace diferencia entre ellos, su propósito central es mostrar las tendencias del pensamiento en el tiempo. (Rubiano, 2005)

\section{Cuadro 3}

Tendencias ideológicas sobre desarrollo rural a través del tiempo

\section{$1950-1960$}

Desarrollo comunitario. Este tema hizo énfasis en la escolaridad, las aptitudes, la salud de los agricultores, promovió la industria artesanal y el desarrollo de las pequeñas explotaciones agrícolas. Se percibe el advenimiento de un sector agrícola moderno que desplaza la idea de sectores tradicionales poco funcionales.

$1960-1970$

Revolución verde. Se asocia con grandes inversiones públicas en infraestructura, investigación y apoyo para la adopción de nuevas tecnologías. Es un enfoque de transformación que busca la modernización: transferencia de tecnología, extensión agrícola, reforma agraria, los campesinos como agentes económicos racionales.

\section{$\mid 1970-1980$}

Desarrollo rural integrado. Se asocia con el progreso de los pequeños productores agropecuarios, se centra en la producción de alimentos más que la producción de cultivos, en los objetivos de distribución más que en los de crecimiento, se establecen política agrícolas estatales. Se consideró la agricultura como un conjunto de recursos, como la veían los productores de cultivos comerciales, pero no se dieron incentivos para aumentar la producción. 
El concepto de territorio desde las ciencias sociales ha sido objeto de polémicas y discusiones. Para algunos, el territorio está concebido como el lugar sagrado donde existen unas tradiciones culturales que implican unas reglas o normas preestablecidas para actuar sobre él, esta es desde luego una visión de territorio ejercida desde una tradición religiosa como es la de los indígenas. Para otros, el territorio es una categoría política que posee un sentido institucional y por lo tanto, es objeto de un ordenamiento específico, determinándolo como una categoría de división político-administrativa la cual está determinada por un río, una cordillera o alguna circunstancia natural que determina un límite político. (Solarte, 2003).

El territorio para el presente trabajo es analizado como un sistema complejo con propósito definido, el cual observa desde una visión integral, los procesos históricos, sociales, culturales, políticos, que conforman una estructura compleja de relaciones hacia el interior y hacia su entorno.

Dependiendo de la visión de mundo desde la cual se configure el territorio este "comienza a adquirir un significado". (Checkland y Scholes, 1994), es decir; la delimitación de un territorio estará dada por un observador, el cual establece una interpretación y le confiriere una estructura y unas características particulares al mismo. Esta configuración se encuentra atribuida al conocimiento basado en la experiencia de dicho observador.

Teniendo en cuenta la visión de mundo y de los observadores, la delimitación de un territorio no puede establecerse simplemente como una organización político administrativa; por el contrario, tal como lo propone Solarte (2003) el territorio establece una compleja red de relaciones abiertas y de difícil delimitación.
Un territorio puede variar en su tamaño o en su estructura dependiendo del objetivo, interés y aprendizajes propios del observador, un centro poblado, una vereda, un municipio, una provincia, un departamento, un país, o un continente, son territorios desde su delimitación, pero cada uno de ellos guarda un conjunto de relaciones propias, que le otorgan una identidad.

El concepto de territorio desde una visión sistémica, se puede entender como una compleja red de relaciones dinámicas, las cuales constantemente se retroalimentan. Dichas relaciones implican diversas dimensiones, concediéndole un carácter multidimensional, donde los distintos componentes conforman el territorio así; los sectores sociales, políticos, económicos, culturales y ambientales, involucran diversos actores, grupos y por supuesto intereses, interactuando en lo rural, potenciando la diversidad social y cultural además de imprimir la necesidad de ofrecer un manejo sostenible a los recursos naturales (Sepúlveda et al, 2001).

El territorio puede presentarse como un holón,es decir, una unidad con propósito definido. El territorio se convierte en un sistema de actividad humana, el cual es susceptible de comparación con su entorno. Sin embargo, para que el territorio logre adaptarse y establecer flujos de energía con el entorno debe retroalimentarse y contener propiedades emergentes, una estructura definida, y procesos de comunicación y control. (Checkland y Scholes, 1994).

La retroalimentación permanente entre el sistema territorio y su entono determina que los actos se puedan reforzar o contrarrestar entre sí, el lenguaje sistémico reconoce una amplia gama de interrelaciones y patrones de cambio, donde los círculos de causalidad forman la realidad y la linealidad es la razón de la fragmentación de 
la misma, el lenguaje modela la percepción, lo que se ve depende que cómo (los propietarios del sistema) se este preparado para verlo, el pensamiento sistémico, el concepto de retroalimentación manifiesta el flujo recíproco de influencia, la influencia es "causa" y "efecto" nunca es una sola dirección (Pereira, 2002). Hay dos tipos de retroalimentación: de refuerzo y de equilibrio (compensadora), "... la primera es un motor de crecimiento cuando se encuentra en un situación de crecimiento pero también puede presentarse un crecimiento en decadencia.

La retroalimentación compensadora opera cuando hay una conducta hacia las metas, si la meta es no moverse la retroalimentación controladora actúa como un paro total, si la meta es moverse la retroalimentación se mueve de acuerdo con las necesidades de la meta la cual es un objetivo explicito (Pereira, 2002).

El sistema territorio trasciende lo espacial, es considerado un producto social e histórico, lo que le confiere una identidad propia, dotado de recursos naturales, determinadas formas de producción, consumo e intercambio y una red de instituciones y formas de organización que se encargan de darle cohesión a todos los elementos. (Sepúlveda et al, 2001). Son las instituciones y organizaciones del territorio las propietarias del sistema. Son quienes lo delimitan y ejercen los mecanismos de comunicación y control del mismo. La participación de los involucrados, de los beneficiarios, de los propietarios-los pobladores rurales- es imprescindible, pues son ellos quienes conocen sus necesidades y posibilidades, son quienes deben decidir el uso de los recursos y sus posibilidades. De manera que las relaciones e interrelaciones que existen entre los sectores (sociales, políticos, económicos, culturales y naturales) y los pobladores se hacen más que evidentes y necesarios para promover desarrollo.

No es desconocido que las delimitaciones políticoadministrativas no siempre están en concordancia con las realidades económicas, sociales, culturales e incluso naturales preestablecidas en los mismos. La diferencia entre el territorio como una organización política delimitada y el territorio como red de relaciones abiertas y de difícil delimitación, radica en que "cuando se explora el territorio como un complejo de relaciones es cuando se entienden las realidades territoriales y sus dinámicas, las cuales obligan a pensar en cada una de las especificidades de las comunidades que habitan tales territorios". (Solarte Guillermo, 2003). De tal forma, que es a través de una visión compartida de propietarios y actores del sistema territorio que este define los tipos y formas de transformación necesarias, resultado por supuesto de las diferentes interpretaciones y visiones del mundo de dichos propietarios sobre el propósito del mismo.

Dichas transformaciones como se mencionó, solo puedenserejercidas porlos propietarios delsistema desde el momento en que comparten una visión de lo que desean. Para realizar modificaciones es necesario observar su territorio en su totalidad, de modo holista. A partir de la comprensión de la interacción directa y permanente de cada actor sobre cada una de las dimensiones (económica, política, social, ambiental) y de que un cambio en alguna de ellas representa un cambio a nivel del territorio, se genera como resultado una fuerte interdependencia de todos los elementos que participan dentro del sistema. Los propietarios "...integrantes de organizaciones laborales, de organizaciones de mercados de insumos o de mercados laborales, adaptándosealascondiciones particulares del territorio han hecho surgir 
asentamientos y formas de organización social, con jerarquías, especializaciones, estructuras de relaciones entre los distintos grupos y códigos de organización política..." (Echeverri y Ribero:1998). De igual forma, la existencia de valores y formas culturales en los propietarios del sistema territorio imprimen a los asentamientos y formas de organización características particulares que se reflejan directamente en el mismo.

El territorio observado como un sistema de actividad humana con propósito definido permite observar las interrelaciones existentes más allá de simples cadenas lineales de causa y efecto. Los procesos de transformación se convierten en tarea de todos y cada uno de los actores o propietarios del sistema, se dan a la tarea de aprender de forma colectiva, para poder generar transformaciones colectivas. No sólo en el sentido de aprendizaje adaptativo, sino que tiene lugar el aprendizaje generativo, el cual se basa en la creatividad lo cual es denominado en el lenguaje sistémico como metanoia: " ... desplazamiento mental o cambio de enfoque, transito de una perspectiva a otra". Metanoia viene literalmente de meta (más allá) y noia (de la mente); trascendencia, y, aunque el catolicismo lo tradujo como "del arrepentimiento", los antiguos gnósticos lo concebían como el despertar de la intuición compartida y del conocimiento directo de lo más elevado (Senge, 1990), Este concepto contiene, para Senge, el significado mas profundo del término aprendizaje. "El aprendizaje generativo solo es posible cuando la gente se esfuerza para alcanzar algo que le concierne profundamente, el aprendizaje generativo es abstracto hasta que la gente se entusiasma con una visión que realmente le interesa alcanzar. Dicha visión compartida del territorio permite el reconocimiento de sus potencialidades desde las diversas dimensiones encontrando las pautas internas que lo hacen competitivo, es la valoración de lo que es común lo que desarrolla una identidad común..." (Senge, 1990)

El enfoque territorial logra superar la visión convencional de participación y compensación a favor de los conceptos de cooperación, autogestión e inclusión económica y social; desde una concepción multidimensional que logra integrar lo ambiental, lo económico y lo político institucional, generando una identidad particular asentada sobre una determinada base de recursos naturales, ciertas formas de producción, consumo e intercambio, armonizada por las instituciones y las formas de organización que operan en él. (Sepúlveda et al: 2001).

Teniendo en cuenta que el territorio ha sido definido como un holón con propósito definido, surge la interrelación entre el territorio y su entorno; donde el territorio influye en el entorno y viceversa, estableciendo una relación reciproca.

La visión territorial del desarrollo rural, permite una mejor comprensión de la complejidad de lo rural, donde la relación entorno natural -historia, establecen una permanente construcción de territorialidad, la cual imprime y exalta la comprensión de la diversidad o heterogeneidad como elementos fundamentales para construir políticas que se ajusten a las realidades territoriales. (Rubiano, 2005) 


\section{La cooperación local y el DESARrollo TERRITORIAL}

\section{$\mathbf{L}$}

os modelos de gestión del desarrollo rural han presentado cambios importantes a lo largo del tiempo, las políticas e iniciativas que se definen y ejecutan desde los niveles centrales, con baja o nula intervención de los actores locales hacen parte del enfoque de gestión vertical de arriba hacia abajo, el cual se transforma a partir de la mitad de los años ochenta en un enfoque que concibe el desarrollo rural como un proceso de abajo hacia arriba, en donde la participación y el empoderamiento de todos los actores juega un papel fundamental en la construcción de desarrollo. Este cambio se hace evidente en Colombia a partir del establecimiento de la Constitución de 1991, la cual establece el traslado de la soberanía de la Nación al pueblo y consagra el Estado Social de Derecho, como el reconocimiento de la función del ciudadano y de la sociedad civil en la formulación, planeación, ejecución, seguimiento, evaluación, control y vigilancia de la gestión pública. Es decir, se establece la participación activa de los ciudadanos en el proceso de toma de decisiones, lo que incluye no solo la elección de los gobernantes, el disfrute de la distribución y goce de los bienes y servicios de la nación, sino también, la participación en la formulación de políticas y su implementación, y en los mecanismos de control sobre la ejecución de la misma.

Estos cambios, han estado fuertemente vinculados a las transformaciones que se registran en el marco institucional, nuevas reglas de juego, nuevas formas de poder, nuevas formas de interrelacionamiento, establecen una transición de las responsabilidadesdelasinstituciones. Esnecesario comprender, que la institucionalidad que aquí se suscribe va más allá, de las instituciones públicas formales, las instituciones se entienden como "... el conjunto de reglas de juego que dominan el espacio público, el espacio de inserción entre los diferentes intereses individuales, en un contexto de interacción colectiva... La institucionalidad es garante de los derechos reconocidos para los miembros de una sociedad y brinda parámetros para el ejercicio y la misma acción privada..." (Echeverri y Ribero, 1998).

La cooperación local, presenta varias nomenclaturas y definiciones, sin embargo, todas estas representan el mismo tipo de gestión, son procesos donde los ciudadanos asumen un papel como socios de su desarrollo, comunidades autónomas, con capacidad de autogestión y autoinstitución. Para el Instituto Interamericano de Cooperación para la Agricultura -IICA-. La cooperación local, emerge como una estrategia que busca la construcción de alianzas entre socios, orientada a la movilización de la voluntad colectiva con el propósito de lograr el bien común. Investigadores de la Universidad Nacional de San Luis (Argentina), establecen la cooperación local como un modelo que comprende actividades relacionadas con el aprovechamiento de los recursos locales expresados en sus potencialidades culturales, institucionales, económicas, sociales y políticas para el desarrollo de sistemas territoriales innovadores y competitivos.

También resalta en la Universidad Nacional de San Luis el destacado trabajo para comprender unidades sistémicas dentro de una aproximación epistemológica que involucra el análisis de 
sistemas históricos en la perspectiva de Immanuel Wallerstein ${ }^{7}$. Estableciendo un aprovechamiento de las habilidades de los actores públicos y privados en la promoción de su articulación, para impulsar procesos de fomento productivo destinados a dinamizar actividades con capacidad de generar riqueza y empleo.

Para el programa Leader Europeo, la cooperación local consiste en la constitución de una red de relaciones y de solidaridad en un territorio, con el fin de valorizar al máximo su potencial y enriquecer las acciones sectoriales con una reflexión transversal, intersectorial. Aparte de las acciones económicas de consumo realizadas, la cooperación local representa una voluntad de construcción o de reconstrucción de un vínculo social, es decir, de una búsqueda de identidad.

Es una estrategia de trabajo colectivo en red, que permite la vinculación comprometida de los ciudadanos transformando las visiones individualistas en iniciativas de beneficio común. Es una propuesta de trabajo que parte de la movilización de recursos propios (capacidades y potencialidades), del trabajo local y sobre todo, del operar de cada habitante en su territorio para hacerlo socio del desarrollo del mismo. (Bejarano, 2004).

\section{La coOperación local y TERRITORIAL EN EL CicLO DE COMPETENCIAS}

$\mathbf{E}$

territorio ha sido definido como un "producto histórico" conformado por un conjunto de tradiciones o ritos, incorporados por la suma de cosmovisiones, lenguajes, formas de relación, valores estructurados alrededor de una moral, de principios éticos, (Echeverri y Ribero, 1998), así como un conjunto de recursos y formas de apropiación particulares, que determinan la forma de organización, de estructuración del territorio, lo cual le imprime un conjunto de capacidades, una identidad, una apropiación por parte de sus habitantes. En resumen, los territorios cuentan con un conjunto de valores, recursos y capacidades con los cuales se desenvuelven y adaptan a su entorno.
Sin embargo, es común encontrar que dichos territorios poseen un conjunto de valores que generalmente inhiben o limitan el desarrollo de su territorio, en donde la baja capacidad de innovación, restringe que se puedan maximizar sus recursos, su manera de interrelacionarse, de tal forma, que no logran encontrar las formas más óptimas para potenciar sus capacidades y establecer vínculos que les permitan mantenerse en constante interacción con su entorno de una forma competitiva, para alcanzar desde luego, un desarrollo sostenible y acorde con las realidades particulares del territorio. (Rubiano, 2005)

\footnotetext{
7 Al respecto se destaca el trabajo del proyecto de Investigación consolidado 4-1-9301 “Tendencias Epistemológicas y Teorías de la subjetividad. Su impacto en las ciencias humanas" dirigido por la profesora Violeta Guyot en la Universidad Nacional de San Luis, Argentina. El grupo orientado por la profesora Guyot acoge las orientaciones en ciencias sociales de I. Wallerstein y de I. Prigogine en sistemas alejados del equilibrio en ciencias naturales.
} 
El proceso de cooperación local, es complejo, requiere de nuevos aprendizajes por parte de los actores, nuevas pautas de comportamiento, nuevas formas de interacción entre los agentes del territorio y entre este y su ambiente, esta condición esta determinada por factores de innovación que permitan o faciliten procesos de aprendizaje. La cooperación local, se convierte en una estrategia de innovación para el desarrollo de aprendizajes colectivos en un territorio, generando cambios estructurales, los cuales se ven reflejados de forma práctica en la "cultura" del territorio, en el conjunto de normas y valores que se comparten por los propietarios del mismo. Son precisamente estos elementos culturales, estas normas y valores, los que determinan nuevas posibilidades, capacidades u oportunidades para el mismo y es a través del aprendizaje de esa cultura, que el territorio logra interactuar de forma flexible y rápida a los cambios dinámicos que se presenten a través de la evolución y de su consecuente adaptación. De hecho; como se ha mencionado, cada vez que se presenta un nuevo aprendizaje consecuencia de un nuevo nivel de conciencia se establece para el territorio un nuevo nivel de evolución y organización, incorporando nuevas competencias para el territorio.

La incorporación de aprendizajes por parte del proceso de Cooperación Local, determina un arreglo instancia el interior del territorio, donde los valores, normas y reglas que se comparten se traducen en instituciones con lógicas acordes con la cultura, con los códigos, símbolos y lenguajes del territorio. Una institucionalidad capaz de reconocer la diversidad y heterogeneidad a partir de una integración que parte de la diferencia y del reconocimiento de las instituciones naturales construidas a través del tiempo por los actores del territorio.
Es a partir de esta autoinstitucionalidad que surge un nuevo arreglo al interior de los territorios; donde la autogestión determina la capacidad de la ciudadanía para construir y decidir sobre lo público, reconfigurando las formas tradicionales, centralistas y clientelistas, transformándolas por una acción que se construye sobre la responsabilidad compartida por todos los agentes territoriales. La autogestión además, define los objetivos de desarrollo, el establecimiento de metas y visiones territoriales, el control social, la cooperación permanente y la exigencia de rendición de cuentas por parte de quienes asumen las responsabilidades (Archivos del equipo de la Misión Rural). La autonomía por su parte, establece el ejercicio de libertad de los individuos y comunidades, confiriéndole la capacidad para decidir por sí mismas, para convertirse en gestoras y protagonistas de su propio desarrollo, transformando las estructuras y relaciones jerárquicas, asistencialistas y paternalistas, donde la acción colectiva se reduce a acciones contestatarias o reivindicativas. Ciudadanos y comunidades autónomos son el objetivo real de la cooperación local, estableciendo con esto una competencia central para el territorio.

La autoinstitucionalidad, la autonomía y la autogestión sólo pueden ser alcanzados por territorios con capacidad de responsabilizarse y asumir el desarrollo por sí mismos, donde se compartan las visiones para asumir los problemas y se planteen soluciones conjuntas, es decir; alcanzar estos tres elementos, hace parte de un estado de transformación que proviene de una estrategia de innovación como lo es la cooperación local, que permita establecer arreglos al interior del territorio y genere aprendizajes organizacionales que faciliten la generación de autonomías (competencia territorial) para lograr desarrollarse efectivamente en un entorno cambiante. 
Figura 7

La couperación íocai y ius cicios de competencias

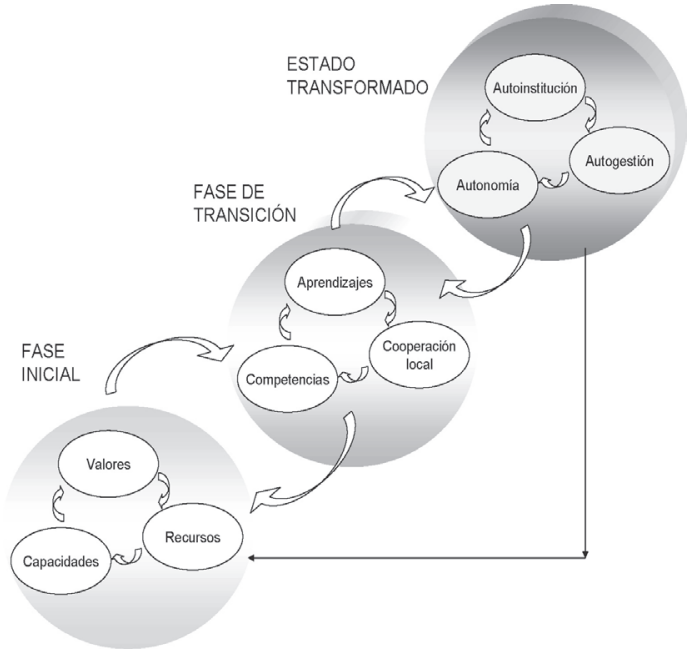

\section{Conclusiones}

En el presente documento se presenta la idea de ciclos de competencias y se propone su empleo creativo en el desarrollo de estrategias de cooperación local.

El territorio se ha señalado como un sistema, el cual se delimita y adquiere una configuración de acuerdo con los propietarios y las visiones de mundo que lo componen. Dicho territorio adquiere a partir de estrategias de innovación como la cooperación local, aprendizajes sociales que le permiten desarrollar competencias para interactuar de forma dinámica con su entorno.

La cooperación local es una estrategia en la que se hace efectiva la participación social en el desarrollo, es un modelo de planificación de abajo hacia arriba que involucra a las organizaciones de la sociedad civil en el diseño de su propio destino y desarrollo, generando un modelo competitivo de largo aliento que promueve la autoinstitución, la autonomía y la autogestión, como elementos que permiten la articulación entre el Estado y la sociedad con el proceso de desarrollo.
La acumulación de aprendizajes sociales, se reflejan en la acumulación de capital social, de donde los valores como la confianza, la solidaridad, la reciprocidad, la cooperación entre otros, refuerzan la generación de nuevas competencias, dando paso a nuevos niveles de evolución del territorio y su mejor adaptación y supervivencia en el entorno.

La cooperación local, siendo una estrategia de innovación que le permite al territorio interactuar y adaptarse mejor ante las condiciones de su entorno, rescata las potencialidades, tradiciones, ritos y elementos que comparten los agentes de un territorio, como elementos facilitadores que se articulan en una visión compartida sobre lo que desean sea su desarrollo. De tal forma, que se convierte en una estrategia que facilita la acumulación continua de aprendizajes, a partir de una historia compartida, de un sentido de pertenencia, de una identidad, de una territorialidad, que conjuga problemas y potencialidades comunes para la construcción conjunta de soluciones. 


\section{Conchusiones}

Ackoff,R . Rediseñando el futuro. México, Limusa, 1994.

Ashby, W.R. An Introduction to Cybernetics, Londres: Fontana. 1956. P. 115.

Bañegil, T, \& Sanguino R. (2003). "Gestión del conocimiento y estrategia". Revista Madrid No. 19 oct- nov.

Bateman, T. y Sell, S. (1996). Management, Building Competitive Advantage. Chicago Third Edition. Irwin, a Times Mirror higher education Group Inc.

Beer, Stafford. Ciencia en la dirección.(1974). La investigación operativa en la empresa. Buenos Aires,Ed. Ateneo.

Beer, S. (1982). Decisión y control: El significado de la investigación de operaciones y la administración cibernética. México. Fondo de Cultura Económica.

Bejarano, A. (2003). "Ciudadanía activa, Guía para la cooperación local."

Beltrán, Jesús Mauricio (1999). Indicadores de gestión. Herramientas para lograr la competitividad. Santafé de Bogotá, 3R Editores.

Bertalanffy L. (1956). General Systems Theory, General Systems. Vol. 1.

Capra, F. (1998). La trama de la vida: una nueva perspectiva de los sistemas vivos. Barcelona, Anagrama, Colección Argumentos.

Cárdenas J. (2003). "Aproximaciones desde los Sistemas Complejos adoptivos al Estudio de la Nueva Ruralidad."

Checkland, P. Scholes,Jim.(1994). La metodología de sistemas suaves en acción. Colección Megabyte. México:Noriega.

Contreras, R. (2000). "Empoderamiento Campesino y Desarrollo Local". Revista Astrual de Ciencias sociales, No 4, Enero.

Dávila, C. (2004). "El Kibbutz como sistema organizacional agrícola." Universidad Nacional de Colombia. Proyecto de grado para optar por el titulo de Ingeniera Agrónoma.

Drucker, P. (1995).The information executives truly need, Hardvard Business Review, JanuaryFebruary. 
Durston,J. (2004). "Capital social: Parte del problema, parte de la solución, su papel en la persistencia y en la superación de la pobreza en América Latina y en el Caribe."

Echeverri, R. (2003). "Economía y Competitividad del Territorio Rural."

Elster J. Inglehart,R. \& Eisler, R. (2003). "Reflexiones Sobre la Investigación en Ciencias Sociales y Estudios Políticos." Universidad Nacional de Colombia. Memorias Seminario Octubre de 2002.

Garzón C. (2000) Sistemas integrados de información para producción. Universidad Nacional. Santafé de Bogotá, Abril.

Goldratt, E. (1993) La Meta. Ed. Ediciones del Castillo, México.

Goldratt, E. (1995). No fue la suerte. México. Ediciones del Castillo.

Hamel G. y Prahalad, C.K. (1996). Las oportunidades del mañana. Revista Gestión. Volumen 1, Número 2. Bogotá, Marzo - Abril 1996. Pag. 62 a 66.

Hamel, G. y Doz, Yves L.(1996). Redes y muñecas rusas. Revista Gestión. Volumen 4, Número 2. Bogotá, Marzo - Abril. Pag. 62 a 66.

Harrington, J. (1998). Mejoramiento de los procesos de la empresa. Santafé de Bogotá. McGraw Hill.

Hamel, G. (2000). La era de la revolución. Revista Gestión, Volumen 5, Número 5. Bogotá, Septiembre - Octubre de 2000. Pág. 70-86.

Hamel, G. (1997) Leading the revolution. Revista Gestión, Book summary, № 4. Bogotá, 2001. HAMEL, Gary. La fórmula de la revolución. Revista Gestión, Volumen 1, Número 2. Bogotá, Mayo - Junio de 1997. Pág. 104 a120.

Kast, F. E. y Rosenzweig, J.E. (1979) Administración de las organizaciones: un enfoque de sistemas. México: Mc Graw Hill.

Koulopoulos, T. Frappaolo, C. (2000) Gerencia del conocimiento. Colección Smart. Bogotá: Mc Graw Hill.

León Medina, E. (2000) Diagnóstico de la Gestión Tecnológica en empresas con procesos biotecnológicos: Sector Alimentos y Bebidas. Bogotá: Universidad Nacional de Colombia.

León Medina, E. et.al. (2002) Creación de Competencias Centrales y Reestructuración de los procesos de Criminalística de Campo. Escuela de Administración de Negocios EAN. Bogotá. 
Leonard-B.- Barton, D. (1995) Wellsprings of knowledge: building and sustaining the sources of innovation. Harvard Business School Press. Boston.

Louart, P. (1994) Gestión de los recursos humanos. Barcelona: Ediciones Gestión 2.000.

Macintosh, A. (1997) Position Paper on Knowledge Management. Artifitial Intelligence Applications Institute, University of Adinburgh.

Martínez C. (1999) Administración de organizaciones productividad y eficacia. Segunda Edición. Editorial Universidad Nacional. Facultad de Ciencias Económicas.

Maslow, A. (1982). The farther reaches of human nature. Viking Press. Palo alto, California.

Mejía, F. (1998) Gestión tecnológica. Dimensiones y perspectivas. Bogotá: Guadalupe Ltda. Bogotá.

Montoya R.I. (1999) Gestión global siglo XXI. Universidad Nacional de Colombia, Maestría en Administración. Santafé de Bogotá.

Montoya R. I. (1999) Gerencia Genética. Universidad Nacional de Colombia, Maestría en Administración. Santafé de Bogotá.

Montoya, I. \& Montoya, A. (2003). "El Direccionamiento estratégico y su Aplicación en los Sistemas Complejos y en la Gerencia Ambiental." Revista INNOVAR, ene- jun, 2003, No.21. Universidad Nacional de Colombia.

Montoya,I. Leon, E. (2004) Los ciclos de generación de competencias y su aplicación en las organizaciones. En: Revista Innovar, Julio - diciembre de 2004, No. 24 p. 9-27.

Morgan, G. (1996) Imágenes de la organización. Grupo Editor Alfa Omega.

Naisbitt, J. Megatrends. (1984) Warner Books Edition, a Warner Comunications Co. Paperback Printing. New York.

Nonaka, I. y Takeuchi, H. (1995) The knowledge-creating company: how Japanese companies create the dynamics of innovation. Oxford University Press. New York.

Observatorio Europeo Leader/Aeid. "Organizar la cooperación local ". Innovación en el medio rural.

Pitt, M, Clarke, K. (1994)Competing on competence: A knowledge perspective on the management of strategic innovation. Technology Analysis \& Strategic Management. Abingdon. 
Prahalad, C.K. (1998) El futuro ha llegado. En: Revista Gestión, Volumen 3, Número 2. Bogotá, Marzo - Abril de 1998. Pág. 16-23.

Ribero P. y Echeverri R.(1998). "Nueva ruralidad, visión del territorio en América Latina y el Caribe"

Rodríguez, J. y Cordero, B.(1999) La Gestión en la Tecnología. Universidad Nacional de Colombia, Facultad de Ingeniería. Santafé de Bogotá.

Rosemberg, M. E-learning. Edit. Mc Graw Hill. New York, 2001. Traducción al Castellano en la misma editorial por Iván Montoya Restrepo.

Roure, J. B.; Moñino, M.; Rodríguez, M.A.; Badal. (1997). La gestión por procesos. Biblioteca IESE de Gestión de Empresas. Barcelona.

Rubiano Navas, M. (2005) La cooperación local como estrategia innovadora para incrementar un aprendizaje colectivo en un territorio. Universidad Nacional de Colombia, Facultad de Agronomía, Tesis de grado.

Senge P. (1992) La quinta disciplina. España: Ediciones Juan Granica.

Sepúlveda S.(2003) “El enfoque territorial del desarrollo rural.” IICA.

Solarte G. (2003). "Cooperación local y ciudadanía".

Tidd, J. (2000) "The competence cycle: Translating knowledge in new processes, products and services" en From knowledge management to strategic competence. Londres: Imperial College Press.

Vollmann, Thomas E, (2005): Planeación y control de la producción administración de la cadena de suministros, México: McGraw Hill. 


\section{PAUTAS PARA LA PRESENTACIÓN DE ARTÍCULOS DESTINADOS A LA REVISTA INSTITUCIONAL DE LA UNIVERSIDAD EAN BOGOTÁ COLOMBIA}

\section{Elementos que debe incluir el artículo}

- Resumen en español y en inglés en el que se expliquen los objetivos específicos y las principales conclusiones del trabajo.

- Palabras claves en español y en inglés.

- Conclusiones o recomendaciones.

- Resumen de hoja de vida en máximo un párrafo, de cada uno de los autores.

- Datos de contacto de todos los autores. (email).

- Carta de autorización de la publicación del artículo, según el formato adjunto. En el caso de autores extranjeros o de otras ciudades, deben enviar el documento escaneado y donde se observen claramente las firmas de los mismos.

\section{Formato}

- Hojas tamaño carta $(21,5 \times 28 \mathrm{~cm}$. $)$.

- Textos escritos en letra Arial 12, en procesador word.

- Espacio doble.

- Extensión mínima 15 cuartillas y máxima 25.

\section{Normas de presentación}

- Notas de pie de página breves con numeración correlativa al texto, pautas de citación correcta y completa bibliografía utilizada para la elaboración del escrito, ordenada alfabéticamente (Protocolo Harvard).

- Codificar figuras, tablas estadísticas, cuadros y gráficos de tal manera que al hacer mención de estos dentro del texto, la relación entre el escrito y el respectivo esquema sea coherente. Los dibujos, gráficos y curvas deben remitirse en tinta negra y en procesador de texto o en paquetes de diseño gráfico. Se deben enviar los archivos originales en los cuales fueron creadas las figuras y los gráficos.

- Concordancia entre los acápites, los textos y los cuadros, tablas, datos y figuras explicativas, adjuntando las series o variables, lo mismo que las notas de pie de página aclarativas, que deben observar la foliación. 
- Utilizar un sistema de nomenclatura que facilite la comprensión del texto.

Nota. Los trabajos presentados suponen que el material es original del autor y no ha sido publicado anteriormente o sometido simultáneamente a otra publicación.

\section{Naturaleza de los artículos}

Teniendo en cuenta que los artículos deben tener un carácter técnico, científico y cultural, el autor del texto puede considerar todos los temas; pero se dará prioridad a los artículos afines a las disciplinas del conocimiento a partir de las cuales la EAN genera sus programas de pregrado y postgrado y sus líneas de investigación.

Los artículos podrán ser resultados de investigaciones, estudios de casos, análisis teóricos donde se evidencien claramente los aportes analíticos del autos, etc.

\section{Sobre los derechos de autor}

- Los autores de artículos que se publiquen en la Revista, tendrán derecho a recibir 10 ejemplares del número de la edición en la que apareció la colaboración. El autor deberá autorizar a la EAN de manera escrita la publicación de su artículo.

- Los artículos publicados en la Revista de la EAN pueden ser reproducidos con fines académicos citando la fuente y el autor.

- Una vez aceptado un artículo para su publicación, el autor o autores transferirán los derechos a la Universidad EAN por medio de la firma de un Acta de sesión de derechos, de acuerdo con lo estipulado por la Dirección Nacional de Derechos de Autor de Colombia.

- En cualquier caso, el Comité Científico y Editorial de la Revista entiende que las opiniones vertidas por los autores son de su exclusiva responsabilidad.

\section{PASOS PARA LA EDICIÓN DE LA REVISTA}

- El editor recibe los artículos y los somete al Comité Editorial de la Revista.

- Los artículos seleccionados por el Comité Editorial serán evaluados por pares (evaluación doblemente ciega) externos quienes emitirán un concepto sobre la eventual publicación del artículo.

- La Universidad EAN se reserva el derecho de publicar o extractar los artículos y no se responsabiliza por el contenido de los mismos. 